\title{
Compressed Air Massage: Repeated Treatment Causes Less Muscle Oedema Than a Single Treatment
}

\begin{abstract}
Compressed air massage is a new treatment modality that has been shown to cause skeletal muscle capillary dilation for up to 24 hours after a single treatment and significantly hastens healing of diabetic ulcers. This study compares the effect of one treatment of a single muscle group, with repeated treatments of several muscle groups.
\end{abstract}

Methods: Four vervet monkeys underwent one, $15 \mathrm{~min}$, treatment of compressed air massage at 1 Bar, to the tibialis anterior muscle and four animals received similar treatment to the whole lower leg on three consecutive days. The tibialis anterior of the treated and untreated limbs was biopsied immediately after the final treatment. Muscle fibre diameters were measured from l $\mu m$ thick toluidine blue stained resin embedded sections using light microscopy and computerized image analysis software.

Results: For treatment of the whole lower limb, the mean fibre diameter increased by $6.0 \%$ from $47.31 \pm 13.4 \mu \mathrm{m}$ (95\%CI:46.47-48.16) in control biopsies to 50.14 $\pm 13.93 \mu \mathrm{m}$ (95\%CI:49.26-51.02) in treated muscle $(p<0.001)$. Treatment of a single muscle showed an increase in diameter of 11.3\%, from 48.21 $\pm 12.68 \mu \mathrm{m}$ (95\% CI:47.31-49.11) to $53.63+14.29 \mu \mathrm{m}(95 \%$ CI:52.61-54.66 ( $p<0.001)$. Treatment of a single muscle caused significantly more oedema than treatment of the whole limb $(p<0.001)$.

Conclusions: Repeated treatment causes skeletal muscle oedema, and this appears to be dose related. Skeletal muscle oedema after three treatments is less than after a single treatment. Further studies on the use of compressed air massage on injured muscle are warranted.

KEY WORDS: COMPRESSED AIR MASSAGE, SKELETAL MYOFIBRES, LIGHT MICROSCOPY, MORPHOMETRY.

\section{INTRODUCTION}

Compressed air massage is a new treatment modality that may be useful in treating skeletal muscle injuries (Mars 2003). Using unheated air, compressed air massage reduces skin temperature, increases skin blood flow during treatment (Mars et al 2005) and causes skeletal muscle capillary dilation for up to 24 hours after a single treatment (Gregory and Mars 2005). When applied to the lower limbs of patients with diabetes, as

\section{CORRESPONDENCE TO:}

Prof Maurice Mars

Dept of TeleHealth

Nelson R Mandela School of Medicine

University of KwaZulu-Natal

Private Bag 7

Congella

4013

Tel: (031) 260-4543

Fax: (031) 260-4737

Email: mars@ukzn.ac.za an additional part of the daily treatment regimen, compressed air massage significantly hastens the healing of diabetic ulcers (Mars and Desai 2005).

Compressed air massage can be varied by using different applicator heads or changing the air pressure. The heads differ in the size, number and configuration of the outlet holes through which the air leaves the applicator head. These range from a single $5 \mathrm{~mm}$ diameter hole to multiple pinholes in a linear array or a single narrow slit. The applicator head with the single $5 \mathrm{~mm}$ hole has been shown to transmit the greatest pressure to subcutaneous tissue and is therefore more likely to cause tissue damage (Mars 2003).

A single, 10 minute treatment, at 1 Bar pressure, using the applicator head with the single $5 \mathrm{~mm}$ diameter hole has been shown to cause ultrastructural changes to skeletal myofibres. These include juxta-nuclear and intermyofibrillar oedema, electron-lucent spaces filled with swollen mitochondria and elements of the sarcoplasmic reticulum (SR) and occasional aggregates of glycogen and other non-contractile organelles in oedematous, sub-sarcolemmal regions, immediately after treatment. Myofibre oedema was significant, with a $17.2 \%$ increase in mean fibre diameter after treatment. $24 \mathrm{hr}$ after treatment, intermyofibrillar oedema was reduced, but SR swelling remained and many fibres were characterised by focal and large areas of myofibrillar disorganisation. Myofibre oedema, while present, was reduced to $5.5 \%$. With the exception of occasional swollen elements of the SR and a single internalised nucleus, myofibres morphology had returned to normal 6 days after treatment (Gregory and Mars 2004). These changes were less frequent and of less severity than those noted after a 10 minute treatment using deep transverse friction (Gregory et al 2003, Gregory and Mars 2004). 
Before conducting clinical trials on the efficacy of compressed air massage on ameliorating muscle injuries, it is necessary to determine the safety and further document the effect that this type of treatment has on normal muscle. As treatment of a single muscle has been shown to improve local blood flow, with sustained capillary dilation 24 hours after treatment, it may be beneficial to treat, not only the injured area, but also the adjacent uninjured muscles to further augment blood flow and possibly lymphatic drainage. Repeated treatment of muscle may however cause more damage than a single treatment. The aim of this study was to examine and compare the effect on skeletal muscle morphology and morphometry, of a single treatment to one muscle group in the lower limb, with repeated treatments of the whole lower limb.

\section{METHODS}

Eight vervet monkeys were studied with the approval of the Ethics and Research Committees of the University of KwaZulu-Natal. The animals were housed in the Biomedical Resource Centre (BRC) and were maintained under the care of the staff of the BRC. The animals were fed ad libitum. Before compressed air massage, anaesthesia and analgesia were achieved by an intramuscular injection of a combination of 50\% Ketamine and 50\% Xylazine, 10 $\mathrm{mg} / \mathrm{kg}$ of vervet monkey body weight. After treatment, the animals were observed during recovery from the anaesthetic and then returned to the holding facility.

\section{Treatment, Muscle Biopsy and Pre- paration}

The hair from ankle to knee was removed from the left and right limbs of each animal with a depilatory to enable observation of any inflammatory reaction and to facilitate treatment. In four animals, in Group 1, treatment was confined to the left anterior compartment. Treatment consisted of one, 15 minute episode of compressed air massage using the three cm, multi-pin-hole applicator head and unheated air at 1 Bar. In four animals in Group 2, the $15 \mathrm{~min}$ treatment was directed over the whole left lower limb from below the knee to the ankle joint. This was repeated on three consecutive days. Immediately after treatment, in Group 1, and after the final treatment in Group 3, the skin was incised longitudinally at the level of the mid calf, the fascia opened and a wedge biopsy of approximately $1 \mathrm{~cm}^{3}$ of tibialis anterior muscle excised from each left limb. To serve as controls, similar biopsies were taken from the right tibialis anterior of each animal within 10 minutes of the final treatment.

To reduce biopsy induced, muscle "supercontraction" artefact which may affect myofibre morphometrics, the entire biopsy was immediately immersed in Karnovsky's fixative (Karnovsky 1965) as per the method of (Olmesdahl et al 1979). After five minutes immersion, the biopsy was examined with a stereomicroscope to determine the alignment of myofibres. Each biopsy was prepared for light microscopy.

\section{Light Microscopic Morphometry}

Biopsies were re-immersed in Karnovsky's fluid for 24 hours prior to dehydration and perfusion and embedding in resin. Care was taken to orientate the tissue to facilitate the cross-sectioning of myofibres. Sections of one $\mu \mathrm{m}$ were cut of the resin embedded tissue, mounted on glass slides and stained with toluidine blue. The sections were examined using a Leitz Biomed light microscope with a X10 objective. Images were collected from the microscope using a Panasonic video-camera (WVCP414E) interfaced with a PC using calibrated Soft Imaging Systems (SIS - version 2.1) software. The morphometric method employed has been previously described (Mars and Gregory 1991). In brief, suitable areas containing transverse/ obliquely sectioned myofibres were selected for morphometric evaluation. The myofibres were considered to be near cylinders and the diameter of fibres taken to be the minimum distance across each cell. A minimum of 117 fibres were measured from each control and experimental specimen.

The mean, standard deviation (SD), and $95 \%$ confidence interval was determined for the fibres within each animal and for each group of animals. The distribution of fibre diameters in each group of specimens was expressed in the form of bar-graphs. Comparisons of

Figure 1: Light micrograph of a $5 \mu \mathrm{m}$ wax embedded toluidine blue stained section showing cross-sectioned myofibres in a treated muscle. The arrows mark the diameters used for morphometery. A necrotic myofibre ( $\mathrm{Nec}$ ) is seen. 10X magnification.

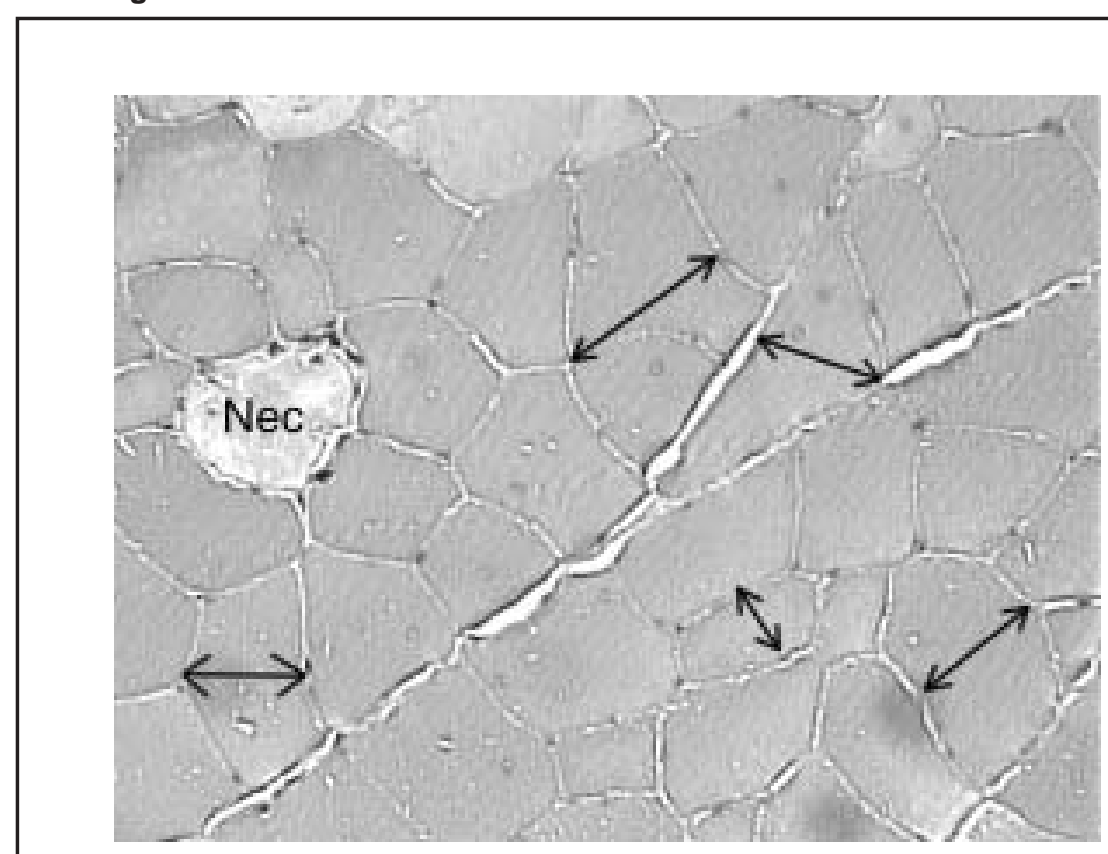


means within and between groups were by analysis of variance using the Kruskal-Wallace ANOVA with post hoc testing using the Tukey Kramer test. Comparison of pooled mean fibre diameters of control and experimental limbs was by unpaired two tailed t-test. Alpha was set at $5 \%$.

\section{RESULTS}

Light Microscopy and Morphometry

The resin embedded tissue was well preserved and the toluidine blue stained sections enabled good resolution of the myofibres using a X10 objective. The working magnification for measurement of fibre diameter on a 14 inch monitor was X350. Most fibres in all specimens were cross-sectioned and appeared as irregular, largely straight sided polygons (Figure 1). While there appeared to be a marginal increase in the space between fibres and groups of fibres in both massaged groups, the fibres did not exhibit any obvious pathomorphology other than a single necrotic fibre in one specimen from an animal in Group 2 (Figure 1).

The averaged morphometric data of fibres obtained from the left and right tibialis anterior of both groups of vervet monkeys are shown in Table 1. Fibre diameters (FD) in the right (control) limbs of the animals in Group 1 ranged from $17.1 \mu \mathrm{m}$ to $92.2 \mu \mathrm{m}$ and in Group 2, from $16.7 \mu \mathrm{m}$ to $82.6 \mu \mathrm{m}$. There was no difference in the fibre diameters of the control biopsies in both groups $(\mathrm{p}=0.158, \mathrm{t}=1.41$, with 1718 degrees of freedom (DF)).

Following the single massage of the anterior compartment, FD in the left limbs of the animals in Group 1 ranged from $19.4 \mu \mathrm{m}$ to $101 \mu \mathrm{m}$. The mean increase in diameter of $5.43 \mu \mathrm{m}$ (95\%CI: 4.06-6.79) was significant $(\mathrm{p}<0.0001, \mathrm{t}=7.80, \mathrm{DF}=1508)$. This constituted a mean increase in FD of $11.3 \%$, with a range from $3.3 \%$ to $18.6 \%$. After three daily massages of the whole lower limb, FD in Group 2 ranged from $16.9 \mu \mathrm{m}$ to $90.5 \mu \mathrm{m}$ and the mean increase in FD of $2.83 \mu \mathrm{m}$ (95\%CI: 1.61-4.05) was significant $(\mathrm{p}<0.0001$, $\mathrm{t}=4,534$, DF $=1918)$. FD increased by $6.0 \%$ with a range of $1.0 \%$ to $16.0 \%$. Treatment of a single muscle caused significantly more myofibre oedema than treatment of the whole limb $(\mathrm{p}<0.001)$.

The marginal distributions of FD in the left and right tibialis anterior in vervet monkeys are shown in Figures 2 and 3. In both groups, the distribution is skewed to fibres of larger diameter after massage.

\section{DISCUSSION}

The main finding of this study is that a single treatment of one muscle group produces significantly more myofibre oedema than treatment of the whole lower limb for three consecutive days. Possible explanations for this observation are, that the response is dose related, repetitive treatment alters local blood flow and or lymphatic drainage, or that treatment sensitises muscle and reduces the effect of barotrauma (Pasupathy and Homer-Vanniasinkam 2005).

When designing the study, it was envisaged that the total dose of compressed air massage per unit area of muscle would be similar in both group 1 , where the anterior compartment was treated once, and in group 2 which received three treatments of the whole lower leg. After the first treatment of the whole lower leg, it would be expected that any barotrauma induced injury would be less than after the single treatment of only the anterior compartment. It might also be expected that repeated

Table 1: Fibre diameter in $\mu \mathrm{m}$, expressed as the mean and one standard deviation (SD), with the $95 \%$ confidence interval $(\mathrm{Cl})$ and percentage change in mean fibre diameter between the control limb and treated limbs in Groups 1 and 2.

\begin{tabular}{|l|c|c|c|c|}
\hline & \multicolumn{2}{|c|}{ Group 1 } & \multicolumn{2}{c|}{ Group 2 } \\
\hline & Control & Treated & Control & Treated \\
\hline $\mathrm{N}=$ & 756 & 754 & 964 & 956 \\
\hline Mean $(\mathrm{SD})$ & $48.21 \pm 12.68$ & $53.63 \pm 14.29$ & $47.31 \pm 13.40$ & $50.14 \pm 13.93$ \\
\hline $95 \% \mathrm{Cl}$ & $47.31-49.11$ & $52.61-54.66$ & $46.47-48.16$ & $49.26-51.02$ \\
\hline$\%$ Change & \multicolumn{2}{|c|}{$11.3 \%$} & \multicolumn{2}{c|}{$6.0 \%$} \\
\hline $\mathrm{P}$ & \multicolumn{2}{|c|}{$<0.0001$} & \multicolumn{2}{c|}{$<0001$} \\
\hline
\end{tabular}

Figure 2: Frequency distribution of fibres from control (Cont. G1) and massaged muscle (Mass. G1) after one treatment of the anterior compartment.

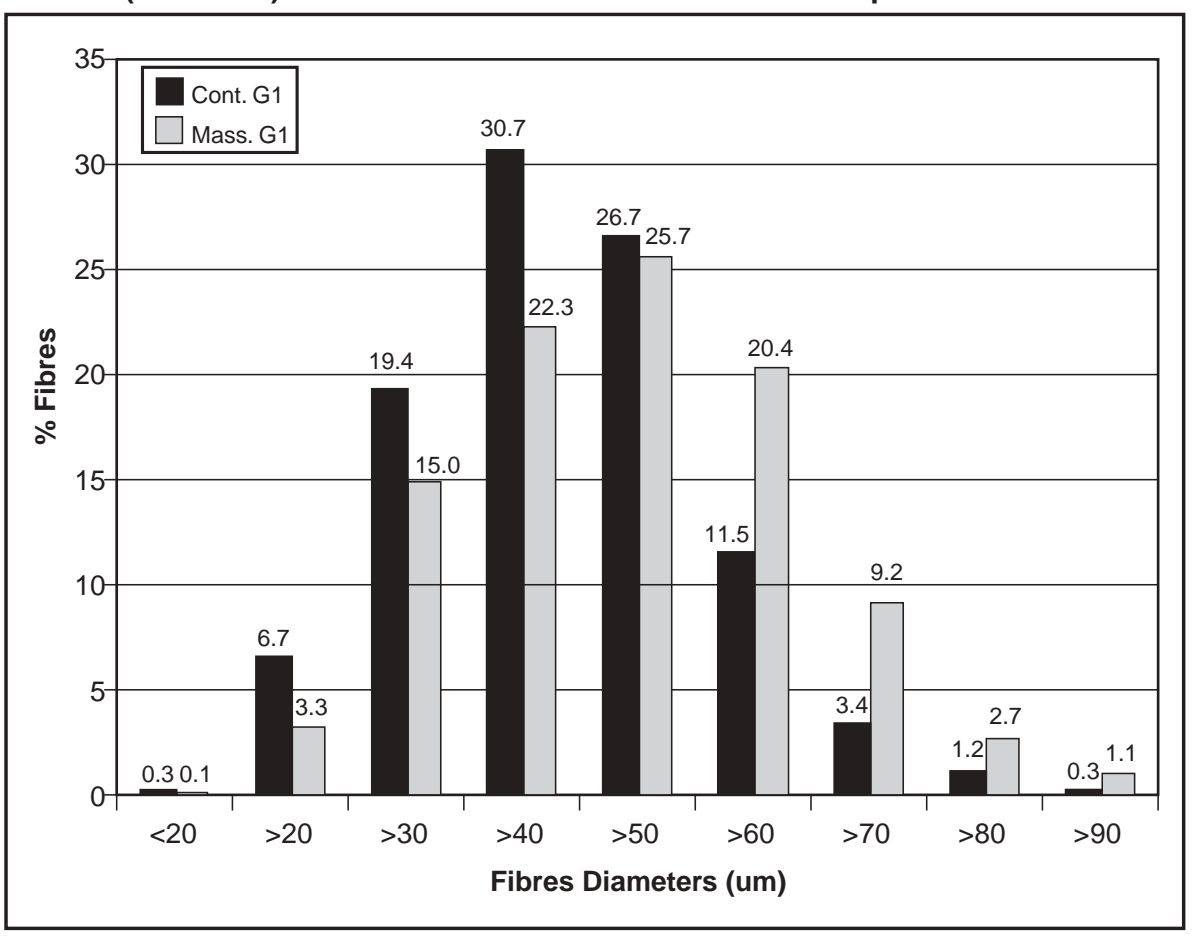


treatment would have an additive effect with a resultant increase in the pathomorphological effects of barotrauma. This did not occur as there was less myofibre oedema after three treatments and no difference in the ultrastructural changes.

The reduction in myofibre oedema after three treatments might also be explained on the basis of capillary dilation after treatment. In a previous study in a rabbit model, capillary cross sectional area increased by $11 \%$ immediately after compressed air massage treatment, increasing to $21 \%, 24$ hours after treatment and returning to normal six days after treatment (Gregory and Mars 2005). Myofibre diameter, which increased by $17.2 \%$ immediately after treatment, reduced to $5.5 \% 24$ hours later (Gregory and Mars 2004), suggesting a link between the increase in circulation and the reduction in myofibre diameter. With repeated treatment, the second and third treatments will have occurred in a setting in which there was already an increase in microcirculation flow. Apart from improving the removal of osmotic metabolites, this may facilitate lymphatic drainage and hasten the resolution of the barotrauma induced myofibre oedema.

Figure 3: Frequency distribution of fibres from control (Cont. G2) and massaged muscle (Mass. G2) after three treatments of the whole lower leg.

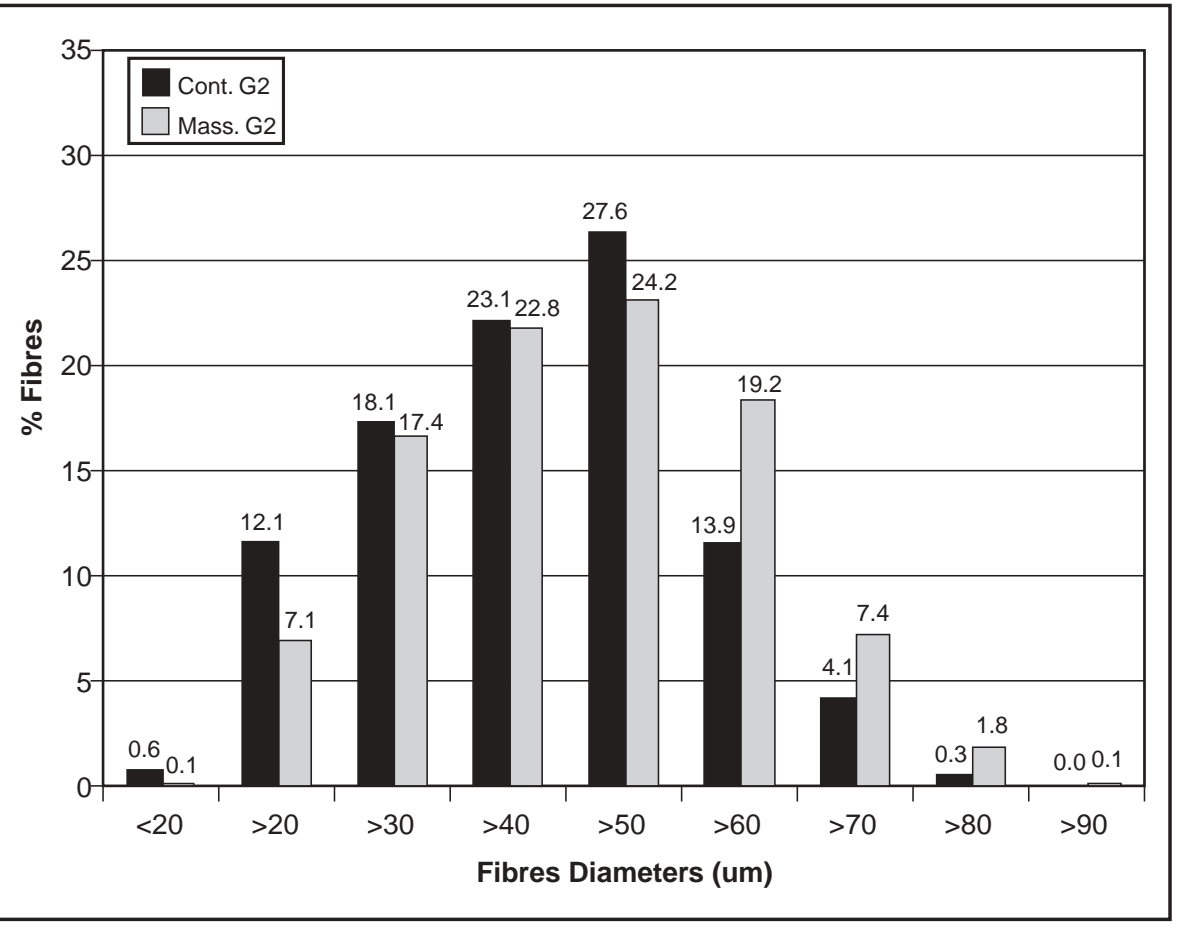
(Mars 2002). Blood flow in capillaries is compromised at relatively low externally applied pressure, with blood flow ceasing in $50 \%$ of capillaries at an external pressure of $12 \mathrm{mmHg}$ (Vollmar et al 1999). It has been shown that ischaemic preconditioning can reduce ischaemic reperfusion injury (Pasupathy and Homer-Vanniasinkam 2005) and it is possible that treatment causes a repetitive ischaemic reperfusion cycle that preconditions the muscle to repeated treatment.

This study has shown that a single treatment of an isolated muscle causes more myofibre oedema than daily treatments of the lower limb for three days. This suggests that repetitive treatment is not associated with increased injury due to an additive effect. In the clinical setting it is suggested that treatment not be localised over the area of muscle injury but rather that treatment should include adjacent untraumatised regions. Further studies are required to determine if treating one muscle group repeatedly is potentially hazardous and or whether repeated treatments reduce the effects.

\section{ACKNOWLEDGEMENT}

This study was supported by a grant from the Medical Research Council of South Africa.

\section{REFERENCES}

Gregory MA, Deane MN, Mars M 2003 Ultrastructural changes in untraumatised rabbit skeletal muscle treated with deep transverse friction. Physiotherapy 89:408-416

Gregory MA, Mars M 2004 The effect of compressed air massage on untraumatised rabbit skeletal muscle - a morphometric and ultrastructural study. South African Journal of Physiotherapy 60:19-27

Gregory MA, Mars M 2005 Compressed air massage causes capillary dilation in untraumatised skeletal muscle: a morphometric and ultrastructural study. Physiotherapy 91:131-137

Karnovsky MJ 1965 A formaldehyde glutaraldehyde fixative of high osmolarity for use in electron microscopy. Journal of Cell Biology 27:127-139

Mars M 2002 Jet therapy - its effect on skin blood flow, muscle pressure and blood parameters. South African Journal of Surgery 40:23

Mars M 2003 Compressed air massage, a new treatment modality? Medicine and Science in Sports and Exercise 35:S92

Mars M, Desai Y 2005 Compressed air massage hastens diabetic ulcer healing. ANZ Journal of Surgery 75:A59

Mars M, Gregory MA 1991 A histometric analysis of skeletal myofibers following 90 min of tourniquet ischemia and reperfusion. Journal of Surgical Research 50:191-195

Mars M, Maharaj SS, Tufts M 2005 The effect of compressed air massage on skin blood flow - a pilot study. Cardiovascular Journal of South Africa 16:208-212

Olmesdahl PJ, Gregory MA, Cameron EWJ 1979 Ultrastructural artefacts in biopsied normal myocardial biopsy in man. Thorax 34:82-90

Pasupathy S, Homer-Vanniasinkam S 2005 Surgical implications of ischemic preconditioning. Archives of Surgery. 140:405-409

Vollmar B, Westermann S, Menger MD 1999 Microvascular response to compartment syndrome-like external pressure elevation: an in vivo fluorescence microscopic study in the hamster striated muscle. Journal of Trauma 46:91-96 